\title{
¿Cuál es el Criterio Utilizado para Incluir algunas Variaciones Anatómicas en Terminologia Anatomica?
}

\author{
What is the Critera Used to Include Certain Anatomical Variations in Terminologia Anatomica?
}

Bélgica Vásquez ${ }^{1} \&$ Mariano del Sol ${ }^{2}$

VÁSQUEZ, B. \& DEL SOL , M. ¿Cuál es el criterio utilizado para incluir variaciones anatómicas en Terminologia Anatomica?. Int. J. Morphol 38(4):1136-1141, 2020.

RESUMEN: Desde la aprobación de la Nomina Anatomica de Basilea (BNA, 1895), Nomina Anatomica de Jena (JNA, 1933) y Nomina Anatomica de Paris (PNA, 1955) y sus posteriores ediciones, siempre fueron incorporados términos de variaciones anatómicas. En las dos primeras ediciones de Terminologia Anatomica (TA) (1998 y 2019), es posible encontrar numerosos términos anatómicos, colocados entre paréntesis, que indican que ellos, de acuerdo a su definición anatómica, no constituyen una normalidad. Cabe preguntarse ¿cuáles fueron los criterios para incorporar estos términos? Conocedores de la enseñanza anatómica, sabemos que normal es un concepto con un fuerte componente estadístico. Siempre se ha señalado que en la normalidad anatómica el criterio más importante es el estadístico, es decir, que la estructura anatómica se encuentre en el mayor porcentaje de la población, en general, sobre el $50 \%$ de los casos. En consecuencia, aquellas estructuras que en número están bajo del promedio estadístico, no deben considerarse normales desde el punto de vista anatómico. El concepto de normalidad en anatomía humana es diferente al concepto de normalidad en un paciente, ya que en este caso normal se considera cuando la persona no está incubando o padeciendo una enfermedad. En este artículo presentamos una serie de términos incorporados a TA y que, desde nuestro punto de vista, no debiesen constar en ella. Por otra parte, indicamos algunos que, con mayor porcentaje estadístico, producto de resultados de exhaustivos trabajos de investigación, no fueron incorporados en dicha Terminología.

PALABRAS CLAVE: Terminologia Anatomica; Variaciones anatómicas; Términos anatómicos.

\section{INTRODUCCIÓN}

Una de las condiciones principales de una terminología anatómica, es que su contenido sea claro y preciso para ser usada sin inconvenientes por quienes desarrollan las diversas disciplinas del área morfológica. A fines del siglo XIX, no existía una nomenclatura morfológica común a todos, era posible observar una infinidad de términos, muchos de los cuales reflejaban su origen y su historia, como también más de una denominación para una misma estructura, plagado de sinónimos, redundancias anatómicas e incluso nombres de estructuras inexistentes. Así, a fines del siglo XIX más de 50 mil términos, solo en anatomía humana, eran utilizados o conocidos en la época.

Conociendo esta realidad, los anatomistas alemanes, en la primera reunión de la Anatomische Gessellschaft en 1987, liderados por el Dr. Wilhem Hiss, iniciaron la preparación de la primera lista de nombres anatómicos, constituida totalmente de términos latinos. Después de un periodo de ocho años fue aprobada el 19 de abril de 1895, constituyéndose en la primera nomenclatura anatómica, conocida como Nomina Anatomica de Basilea (BNA). Casi el $90 \%$ de los términos utilizados en aquél entonces fueron suprimidos, por tratarse de sinónimos, términos incorrectos, entre otros. Por supuesto, hubo imperfecciones, fallas lingüísticas, términos mal utilizados, estructuras que quedaron sin nombre o incorporación de términos que, por su frecuencia, no deberían haber sido incluidos en la Nomina Anatomica, ya que se postulaba que solo estarían en la nomenclatura aquellos términos que representasen la normalidad anatómica.

Hubo muchas revisiones posteriores, modificaciones e incorporaciones de nuevos términos. Este hecho se ve reflejado en la Nomina Anatomica de Paris (PNA), publicada en 1955, la cual constaba de 5640 términos de los cuales 4286 provenían de $B N A$.

\footnotetext{
${ }^{1}$ Universidad de Tarapacá, Chile.

${ }^{2}$ Centro de Excelencia en Estudios Morfológicos y Quirúrgicos (CEMyQ), Universidad de La Frontera, Chile.
} 
Uno de los aspectos que nos llamó la atención en las diferentes nomenclaturas anatómicas posteriores, ha sido la incorporación de términos anatómicos que constituyen las denominadas variaciones anatómicas. Es posible observar estas variaciones anatómicas en $P N A$, la cual fue revisada por el Comité Internacional de Nomenclatura Anatómica designado durante el V Congreso Internacional de Anatomía realizado en Oxford en 1950. Las siguientes ediciones de la Nomina Anatomica, y luego las dos ediciones de Terminologia Anatomica (TA), han incorporado cada vez más términos que, en algunos casos, no llegan a representar más allá del $4 \%$.

Por lo anterior, cabe preguntarse ¿cuál ha sido y es el criterio para incorporar términos anatómicos que son variaciones anatómicas? y ¿cuál es la justificación para no incorporar términos anatómicos que, aunque son consideradas variaciones anatómicas, están presentes en alrededor del $30 \%$ de los casos? Las respuestas a estas preguntas no se encuentran fácilmente, ya que no existe una explicación clara en las diferentes nomenclaturas oficiales que señale cuáles son los requisitos de inclusión o exclusión de términos anatómicos.

El objetivo de este trabajo fue presentar una serie de términos incorporados a la TA y que, desde nuestro punto de vista, no debiesen constar en ella. Por otra parte, indicar algunos que, con mayor porcentaje estadístico, producto de resultados de exhaustivos trabajos de investigación, no fueron incorporados en dicha Terminología.

\section{MATERIAL Y MÉTODO}

Para plantear el problema y la discusión posterior, revisamos algunos términos incluido en PNA de 1955 y en TA de 2019.

Nuestro trabajo se limitó solo al análisis de los términos contemplados en los capítulos de Osteologia, Syndesmologia y Myologia de PNA y en la Parte II de TA, correspondiente a Systemata musculoskeletalia con sus capítulos de Ossa, Juncturae y Systema musculare.

Un aspecto importante a considerar es que PNA señala algunas indicaciones orientadoras con respecto al uso de signos de puntuación y resaltes tipográficos: los paréntesis ovales ( ) designan variaciones anatómicas, los corchetes [ ] indican los términos dados como una opción oficial o para adiciones explicativas y las cursivas se usan para términos ontogenéticos.

\section{RESULTADOS}

Se constató que, en la segunda edición de $T A$, los términos relacionados con el capítulo de osteología se han más que triplicado en número. El $72 \%$ de estos términos pertenecen a los huesos de la cabeza, entre los cuales también se incluyen términos formados por dos huesos como el foramen esfenotemporal visible en la base del cráneo y el foramen craneoorbital de la órbita ósea.

En la Tabla I, se encuentran los términos de osteología en PNA y huesos en TA, considerados como una variación anatómica.

Las suturas frontal y escamosamastoidea son los dos términos considerados variaciones en PNA, sin embargo, en $T A$ otras suturas también fueron consideradas, como sincondrosis y ligamentos. Estos resultados se pueden observar en la Tabla II, que incluyen los términos de Sindesmología en PNA y articulaciones (junturas) en TA.

Los términos de Miología en PNA y Sistema muscular en $T A$ son bastante dispares en cuanto a número. PNA considera tres músculos y sólo uno de ellos, el músculo esternal, se encuentra en TA. En cambio, TA ha incorporado numeroso otros músculos, algunos de ellos sin utilizar el prefijo músculo, regla implementada por $P N A$ que dice relación a que todos los músculos deben tener el nombre genérico de músculo, excepto el diafragma y platisma. En la Tabla III, se encuentran los términos de Miología en PNA y Sistema muscular en TA considerados como variación.

\section{DISCUSIÓN}

En numerosos estudios se ha demostrado la presencia de estructuras anatómicas que constituyen variaciones anatómicas y que, por su porcentaje, algunas debieran estar o no incluidas en TA. Así ocurre, por ejemplo, con la Fabella (del latín, pequeño frijol), hueso que se encuentra asociado al tendón de la cabeza lateral del músculo gastrocnemio. $\mathrm{Al}$ respecto, un estudio realizado por Ortega \& Olave (2018) en 200 radiografías de individuos de ambos sexos, observaron la presencia de la fabela en el 37,5\% de los casos. De éstos, $88 \%$ pertenecían al sexo femenino y $12 \%$ al sexo masculino. Berthaume \& Bull (2020), en un metaánalisis señalaron que la fabela se encuentra en personas mayores y con mayor frecuencia en hombres que en mujeres. Este hueso, según este estudio, está más presente en individuos 
Tabla I. Términos de osteología en Nomina Anatomica (PNA) y huesos en Terminologia Anatomica, considerados como variaciones anatómicas.

\begin{tabular}{|c|c|c|}
\hline Nomina Anatomica (PNA, 1955) & Terminologia Anatomica (2019) & Explicaciones de $P N A, 1955$ y $T A, 2019$ \\
\hline \multirow[t]{2}{*}{ Ossa suprasternalia } & Ossa suprasternalia & \\
\hline & Foramen sphenotemporale & \\
\hline \multicolumn{3}{|l|}{ Os interparietale } \\
\hline & Incisura supraorbitalis & \\
\hline & Incisura frontalis & \\
\hline & Foramen frontale & \\
\hline & Spina trochearis; Spina suprameatica & \\
\hline Crista occipitalis externa & Crista occipitalis externa & $\begin{array}{l}\text { Cresta occipital externa. Como una variación ocasional, la } \\
\text { protuberancia occipital externa está representada por una cresta } \\
\text { de hueso. [PNA, 1955]. }\end{array}$ \\
\hline Var. Crista occipitalis interna & Crista occipitalis interna & \\
\hline \multirow[t]{7}{*}{ Processus paramastoideus } & Processus paramastoideus & \\
\hline & Fossa vermiana & \\
\hline & Processus clinoideus medius & \\
\hline & Canalis craniopharyngeus & \\
\hline & Foramen caroticoclinoideum & \\
\hline & Foramen venosum & El foramen de Vesalio [NA4, 1977]. \\
\hline & Foramen petrosum & \\
\hline \multirow[t]{4}{*}{ Spina supra meatum } & Spina suprameatalis & \\
\hline & Concha nasalis suprema & La concha nasal suprema es solamente ocasionalmente vista. \\
\hline & Os japonicum & $\begin{array}{l}\text { Esta variante cigomática bipartita se encuentra en } \\
\text { aproximadamente en } 4 \% \text {. }\end{array}$ \\
\hline & Tuberculum marginale & \\
\hline \multicolumn{3}{|l|}{ Spina trochlearis } \\
\hline \multicolumn{3}{|l|}{ Sutura frontalis } \\
\hline \multicolumn{3}{|l|}{ Concha nasalis suprema } \\
\hline \multicolumn{3}{|l|}{ Tuberculum marginale } \\
\hline \multicolumn{3}{|l|}{ Tuberositas masseterica } \\
\hline \multicolumn{3}{|l|}{ Tuberositas pterygoidea } \\
\hline \multirow[t]{7}{*}{ Ossa suturarum } & Os suturale & \\
\hline & Os interparietale; Os incae & \\
\hline & Premaxilla; Praemaxilla; Os incisivum & $\begin{array}{l}\text { La aparición de un os incisivo o sutura incisiva en la } \\
\text { humanidad es inusual. [NA4, 1977]. }\end{array}$ \\
\hline & Symphysis mandibulae; Symphysis menti & \\
\hline & Torus mandibularis & $\begin{array}{l}\text { Una prominencia en el cara lingual de la mandíbula en la base } \\
\text { de su parte alveolar, adyacente a } 1 \text { os dientes postcaninos. } \\
\text { [NA4, 1977]. }\end{array}$ \\
\hline & Tuberositas masseterica & \\
\hline & Tuberositas pterigoidea & \\
\hline \multirow[t]{5}{*}{ Torus palatinus } & Torus palatinus & \\
\hline & Foramen cranioorbitale & Pertenece a la órbita. \\
\hline & Canalis arteriae vertebralis & \\
\hline & $\begin{array}{l}\text { Processus uncinatus vertebrae thoracicae } \\
\text { primae } \\
\text { Costa cervicalis }\end{array}$ & \\
\hline & Costa lumbalis & \\
\hline Processus supracondylaris & Processus supracondylaris & \\
\hline Os centrale & Os centrale & \\
\hline $\begin{array}{l}\text { Tuberculum obturatorium } \\
\text { posterius }\end{array}$ & Tuberculum obturatorium posterius & \\
\hline \multirow[t]{3}{*}{ Trochanter tertius } & Trochanter tertius & \\
\hline & Fabella & \\
\hline & Cyamellus & \\
\hline Os trigonum & Os trigonum & \\
\hline
\end{tabular}

de Asia, seguidos de Oceanía, América del Sur, Europa, Medio Oriente y América del Norte. Se determinó que una de cada tres rodillas tiene fabela. Señalan además que, en todo el mundo, un promedio de $36,8 \%$ de las rodillas tiene fabelas osificadas detectable por disección. Entonces ¿por qué este hueso no está incluido en TA? Lo avalan más de 65 investigaciones, en más de 21626 rodillas estudiadas.
Otro ejemplo, es la sutura frontal, la cual es generalmente transitoria y une las mitades derecha e izquierda del hueso frontal. Este término se encuentra incluido entre paréntesis en PNA (en Osteologi y en Syndesmologi), la cual señala, que la parte más baja de la sutura a menudo persiste en el adulto. En TA este término solo se encuentra entre paréntesis en Juncturae y se le agregó el calificativo 
Tabla II. Términos de sisdesmología en Nomina Anatomica (PNA) y articulaciones (junturas) en Terminologia Anatomica, considerados como variaciones anatómicas.

\begin{tabular}{|c|c|c|}
\hline Nomina Anatomica $(P N A, 1955)$ & Terminologia Anatomica (2019) & Explicaciones de $P N A, 1955$ y $T A, 2019$ \\
\hline \multicolumn{3}{|l|}{ Sutura frontalis } \\
\hline & Sutura frontalis persistens; Sutura & $\begin{array}{l}\text { Sutura frontal persistente. [Sutura metó pica]. La sutura } \\
\text { frontal se denomina sutura generalmente transi toria entre }\end{array}$ \\
\hline & & $\begin{array}{l}\text { las mitades derecha e izquierda del hueso frontal. La } \\
\text { parte más baja de la sutura a menudo persiste en el cráneo } \\
\text { adulto. [PNA, 1955] Esto indica la persistencia de la } \\
\text { sutura generalmente transitoria entre las mitades de } \\
\text { hueso frontal. Si persiste, a menudo se denomina Sutura } \\
\text { metópica, por lo que este término se ha agregado como } \\
\text { una alternativa oficial. [NA2, 1961]. }\end{array}$ \\
\hline & $\begin{array}{l}\text { Sutura occipitalos accessoria; } \text { Sutura } \\
\text { mendosa }\end{array}$ & \\
\hline \multirow[t]{10}{*}{ Sutura squamosomastoidea } & Sutura squamomastoidea & \\
\hline & Sutura incisiva & $\begin{array}{l}\text { La aparición de un hueso incisivo y sutura incisiva en la } \\
\text { humanidad es inusual. }\end{array}$ \\
\hline & Synchondrosis intraoccipitalis posterior & \\
\hline & Synchondrosis intraoccipitalis anterior & \\
\hline & Ligamentum atlantooccipitale anterius & \\
\hline & Ligamentum occipitale transversum & $\begin{array}{l}\text { El ligamento occipital transversal se encuentra en } \\
\text { aproximadamente el } 44 \% \text {, con frecuencia uniéndose con } \\
\text { el ligamentoalar y diente. }\end{array}$ \\
\hline & Synchondrosis manubriosternalis & $\begin{array}{l}\text { Esta articulación es al principio una sincondrosis y luego } \\
\text { [en la vida] una sínfis is. [NA4, 1977]. }\end{array}$ \\
\hline & Ligamentum transversum inferius & \\
\hline & scapulae & \\
\hline & Ligamentum radiocapitatum & \\
\hline
\end{tabular}

de persistens, por persistir, recibiendo también como sinónimo latino el término Sutura metopica, por que «a menudo se la denomina así». Al respecto, un estudio realizado por del Sol et al., (1989b) en 400 cráneos de individuos brasileños, observaron que la sutura metópica o frontal incompleta estaba presente en el $28,75 \%$ de los cráneos y el metopismo en el 2,75\% de los cráneos. Por su parte, Ajmani et al. (1983) estudiaron la incidencia de la sutura metópica en 206 cráneos de individuos adultos nigerianos, reportando que la sutura metópica (completa o incompleta) estaba presente en el 34,97 \% de los cráneos y el metopismo en el 3,4\% de los casos. Khamanarong et al. (2015), estudiaron 706 cráneos secos (481 hombres y 225 mujeres) tailandeses adultos, señalando que en el 7,51\% estaba presente la sutura metópica. Un estudio interesante sobre el metopismo lo realizó Eroglu (2008) quien investigó la variación de la frecuencia del metopismo en los períodos históricos, en 487 individuos adultos de 12 poblaciones diferentes de Anatolia Antigua, que databan de varios períodos de la historia, que van desde el Neolítico hasta el primer cuarto del siglo XX. Se reveló que la frecuencia del metopismo mostró un aumento relativo en los períodos de tiempo en Anatolia después del período neolítico, con la excepción de la población Cevizcioglu Çiftligi. Determinó que la frecuencia del metopismo en la antigua Anatolia tenía un rango de distribución de 3,3 a 14,9 $\%$. En base a lo anterior, el término sutura frontal colocada entre paréntesis en PNA y TA estaría correcta. Sin embargo, no hay que olvidar que esta sutura está presente en el $100 \%$ de los cráneos de los recién nacidos y en los niños pequeños hasta los diez años de edad, persistiendo incluso en la adultez. Quizás sea necesario la creación de una Terminologia Anatomica dedicada al recién nacido y al niño, tal como existe una Terminologia Embryologica.

Cabe preguntarse por qué el Os japonicum o variante bipartita del hueso cigomático fue incorporado a la $T A$ no superando el $4 \%$. Así como este ejemplo, existen varios otros términos de huesos que debieran ser revisados por la FIPAT e incluidos o retirados de la $T A$.

Por otra parte, si se revisan los términos de Miología en PNA y Sistema muscular en TA, solo los músculos esternal y transverso de la nuca de PNA están considerados en $T A$, sin embargo, 13 nuevos términos de variaciones musculares se han adicionado a $T A$, de cuales 4 de ellos tienen un sinónimo en latín, uno tiene dos sinónimos y otro de ellos tiene tres sinónimos en latín, lo que contradice una de las más importantes recomendaciones de la BNA, JNA y PNA y $T A$ de denominar a una estructura con un solo término.

Oliveira et al. (2017) realizaron una revisión sistemática de la presencia del músculo esternal en artículos publicados entre 1867 y 2016, concluyendo que este músculo está presente entre el 3 a $8 \%$ de la población en general, 
Tabla III. Términos de Miología en Nomina Anatomica (PNA) y Sistema muscular en Terminologia Anatomica, considerados como variaciones anatómicas.

\begin{tabular}{|c|c|c|}
\hline Nomina Anatomica $(P N A, 1955)$ & Terminologia Anatomica (TA) (2019) & Explicaciones de $P N A, 1955$ y $T A, 2019$ \\
\hline & Musculus incisurae terminalis & \\
\hline & Musculus pterygoideus proprius & \\
\hline & Musculus scalenus minimus & \\
\hline & Levator glandulae thyreoideae; Musculus & \\
\hline & levator glandulae thyroideae & \\
\hline \multirow[t]{3}{*}{ M. transversus nuchae } & Musculus transversus nuchae & \\
\hline & $\begin{array}{l}\text { Rotatores lumborum; Musculi rotatores } \\
\text { lumborum }\end{array}$ & $\begin{array}{l}\text { Estos y los siguientes músculos pequeños de la espalda se } \\
\text { han expandido ligeramente. [PNA, 1955] Estos y los } \\
\text { siguientes múscul os pequeños de la espalda se han } \\
\text { subdividido en grupos: cervical, torácico, lumbar, etc. } \\
\text { [NA2, 1961] Los músculos rotatores generalmente se } \\
\text { describen como largos y cortos. }\end{array}$ \\
\hline & $\begin{array}{l}\text { Rotatores colli; } \text { Rotatores cervicis; } \\
\text { Musculi rotatores cervicis; } \text { Musculi } \\
\text { rotatores colli }\end{array}$ & \\
\hline \multirow[t]{3}{*}{ M. sternalis } & Musculus sternalis & \\
\hline & $\begin{array}{l}\text { Pars abdominalis musculi pectoralis } \\
\text { majoris }\end{array}$ & \\
\hline & Musculus interosseus palmaris pollicis & $\begin{array}{l}\text { La cabeza profunda del músculo flexor corto del pulgar, } \\
\text { según algunas autoridades, no debe equipararse con el } \\
\text { músculo interóseo palmar polimérico (Adductor } \\
\text { acccessorius pollicis); pero esta controversia no está del } \\
\text { todo aclarada. }\end{array}$ \\
\hline \multicolumn{3}{|l|}{ M. sacrococcygeus ventralis } \\
\hline \multicolumn{3}{|l|}{ M. sacrococcygeus dorsalis } \\
\hline & Psoas minor; Musculus minor psoae & \\
\hline & Caput tertium musculi recti femoris & $\begin{array}{l}\text { Una cabeza adicional que se adhiere a la cara anterior del } \\
\text { trocánter mayor.iExiste una tercera cabeza del músculo } \\
\text { recto femoral? Tubbs et al., Folia Morphol., 65:377-80, } \\
2006 \text {. }\end{array}$ \\
\hline & $\begin{array}{l}\text { Adductor minimus; Musculus adductor } \\
\text { minimus }\end{array}$ & $\begin{array}{l}\text { El aductor mínimo representa las fibras superiores del } \\
\text { aductor máximo y no un músculo separado distinto. }\end{array}$ \\
\hline & $\begin{array}{l}\text { Abductor metatarsi quinti; Musculus } \\
\text { abductor metatarsi quinti } \\
\text { Musculus opponens digiti minimi pedis }\end{array}$ & \\
\hline B. subcutanea acromialis & Bursa subcutanea acromialis & \\
\hline B. m. coracobrachialis & Bursa musculi coracobrachialis & \\
\hline B. intratendinea olecrani & Bursa intratendinea olecrani & \\
\hline B. cubitalis interossea & Bursa cubitalis interossea & \\
\hline B. iliopectinea & Bursa iliopectinea & \\
\hline B. prepatellaris subfascialis & Bursa subfascialis subfascialis & \\
\hline B. subtendinea prepatellaris & Bursa subtendinea prepatellaris & \\
\hline
\end{tabular}

siendo su presencia menor entre los europeos $(4,4 \%)$ y mayor entre los africanos $(8,4 \%)$ y asiáticos $(11,5 \%)$. Estudios de Nakano citado por Taniguchi \& Tochihara (1932) reportaron la presencia del músculo esternal en un $21,9 \%$ en individuos chinos y Sakima citado por Fukuyama (1940) reportaron la presencia de este músculo en un $23,5 \%$ en esta misma población. Recientemente Duque et al. (2019) reportaron en una población colombiana la presencia del músculo esternal en el 2,94\% de la muestra. Entonces ¿cuál será el mínimo de presencia de una estructura para ser incorporado como un término anatómico?
Por otra parte, también a manera de ejemplo, se ha colocado entre paréntesis al músculo psoas menor, es decir pasó a constituir una variación anatómica ¿desde cuándo ocurrió esta situación? Todos los textos anatómicos siguen considerando al músculo psoas menor como presente de manera normal. Guerra et al. (2012), señalaron que la anatomía del músculo psoas menor, en los seres humanos ha sido a menudo vinculada con cuestiones de orden étnicoraciales. En diversos estudios se reporta la alta frecuencia del músculo psoas menor, así en población indú está sobre el 30 \% (Joshi et al., 2010; Ojha et al., 2016), en brasileños 
59\% (Farías et al., 2012) y en un estudio de 22 fetos encontraron 8/11 en el sexo masculino y 5/11 en el sexo femenino (Guerra et al.). Incluso se reportan cifras más elevadas, 60 $\%$ en búlgaros (Dragieva et al., 2018) $65 \%$ en americanos (Neumann \& Garceau, 2015), entre otros estudios.

En conclusión la $T A$ incorpora términos que constituyen variaciones anatómicas de muy baja frecuencia y no incluye términos anatómicos que por su frecuencia debiesen ser incorporados, ya sea como estructuras normales (fabela, músculo psoas menor) y otras como variaciones anatómicas.

VÁSQUEZ, B. \& DEL SOL, M. What is the critera used to include certain anatomical variations in Terminologia Anatomica? Int. J. Morphol 38(4):1136-1141, 2020.

SUMMARY: Traditionally since their approval, the terms of anatomical variations have been incorporated in the Basle Nomina Anatomica (BNA, 1895), Jena Nomina Anatomica (JNA, 1933 ) and Paris Nomina Anatomica (PNA, 1955). In the first two editions of Terminologia Anatomica (TA) (1998 and 2019), numerous anatomical terms, can be found in parenthesis, which according to their anatomical definition, are indicative that they do not constitute normal standards. Therefore, it is worthwhile inquiring what criteria was used at the time these terms were incorporated. Experience and knowledge in the anatomical teaching field, has asserted that 'normal' is a concept with a strong statistical component. In anatomical normal standards, it has been noted that the statistical criterion is most important. That is to say that the anatomical structure is found in the highest percentage of the population, generally in over $50 \%$ of cases. Consequently, structures found below the statistical average in number should not be considered anatomically normal. The concept of normality in human anatomy is different from that in a patient, given that normal in that case is considered when the person is not incubating or suffering from a disease. In this article we present a series of terms incorporated into $T A$ which, considering our point of view, should not be part of the TA. Nevertheless, as a result of exhaustive research, certain terms with higher statistical percentages are emphasized which were not incorporated in the Terminology.

KEY WORDS: Terminologia Anatomica; Anatomical variations; Anatomical terms.

\section{REFERENCIAS BIBLIOGRÁFICAS}

Ajmani, M. L.; Mittal, R. K. \& Jain, S. P. Incidence of the metopic suture in adult Nigerian skulls. J. Anat., 137(1):177-83, 1983.

Berthaume, M. A. \& Bull, A. M. J. Human biological variation in sesamoid bone prevalence: the curious case of the fabella. J. Anat., 236:228-42, 2020.

Comité International de la Nomenclature Anatomique. Nomina Anatomica. Imprimé à titre de publication privée, Londres, 1955. del Sol, M.; Binvignat, O. \& De Angelis, M. Padrões de distribuição da incisura e forame supra-orbitais. Arq. Bras. Oftal., 52:208-10, 1989a.

del Sol, M.; Binvignat, O.; Bolini, P. D. \& Prates, J. C. Metopismo no indivíduo brasileiro. Rev. Paul. Med., 107:105-7, 1989b.

Dragieva, P.; Zaharieva, M.; Kozhuharov, Y.; Markov, K. \& Stoyanov, G. Psoas Minor Muscle: A Cadaveric Morphometric Study. Cureus, 10(4):e2447, 2018.

Duque, P. J. E.; Barco-Ríos, J.; Vélez-García, J. F. Incidence of sternalis muscle in the Caldas population (Colombia): Anatomical variations. Int. J. Morphol., 37(4):1342-6, 2019.

Eroglu, S. The frequency of metopism in Anatolian populations dated from the Neolithic to the first quarter of the 20th century. Clin. Anat., 21: 471-8, 2008.

Farias, M. C. G.; Oliveira, B. D. R.; Rocha, T. D. S. \& Caiaffo, V. Morphological and morphometric analysis of psoas minor muscle in cadavers. J. Morphol Sci., 9:202-5, 2012.

Fukuyama, U. Der Musculus sternalis bei den Nordchinesen. Okajimas Folia Anat. Jap., 19:69-92, 1940.

Guerra, D. R.; Reis, F. P.; Bastos, A. A.; Brito, C. J.; Silva, R. J. S. \& Aragão, J. A. Anatomical study on the psoas minor muscle in human fetuses. Int. J. Morphol., 30(1):136-9, 2012.

Joshi, S. D.; Joshi, S. S.; Dandekar, U. K. \& Daini, S. R. Morphology of psoas minor and psoas accessorius. J. Anat. Soc. India, 59:31-4, 2010

Khamanarong, K.; Tuamsuk, P.; Woraputtaporn, W.; Namking, M.; Sawatpanich, T.; Toomsan, Y. \& Iamsaard, S. Incidence of metopism in adult Thai skulls. Int. J. Morphol., 33(1):51-54, 2015.

Neumann, D. A. \& Garceau, L. R. A proposed novel function of the psoas minor revealed through cadaver dissection. Clin Anat., 28:243-52, 2015.

Ojha, P.; Prakash, S. \& Jain, A. Morphology of psoas minor muscle- a cadaveric study. Int. J. Curr. Res., 8:35-9, 2016.

Ortega, M. \& Olave, E. Presencia, localización y biometría de la fabela en individuos chilenos: Estudio radiológico. Int. J. Morphol., 36(1):358$61,2018$.

Taniguchi, T. \& Tochihara, J. Studies on sternalis muscle in Japanese, with special references to fetus and anencephalon on Japanese. Acta Anat. Japan, 7:1232-49, 1932.

\section{Dirección para correspondencia: \\ Dra. Bélgica Vásquez \\ Facultad de Ciencias de la Salud \\ Universidad de Tarapacá \\ Arica \\ CHILE}

Email: bvasquezp@uta.cl

Recibido : 26-01-2020

Aceptado: 18-03-2020 